\title{
Targeting Optimal Metabolic Parameters in Type 1 Diabetes Mellitus and Coeliac Disease: An Extra Challenge
}

\author{
Clifford Kiat',2, Thomas Cotter ${ }^{1,}$ Sean Dinneen ${ }^{1}$, Esther S. O'Sullivan1 \\ ${ }^{1}$ Department of Endocrinology and Diabetes Mellitus, ${ }^{2}$ Department of Gastroenterology, Galway University \\ Hospitals
}

\begin{abstract}
Introduction GFD added to a diabetic dietary regimen imposes practical limitations and leads to considerable restrictions in patients' lifestyles. Prevalence of $C D$ in the general population is approximately $1 \%$. In patients with T1DM, the prevalence of $C D$ is higher with studies reporting rates between $0.6 \%$ to $16.4 \%{ }^{1}$ Gluten free diet (GFD)(the cornerstone of managing coeliac disease) imposes practical limitations in dietary options. Many of the GFD have a high glycemic index potentially making glyacemic targets more difficult to achieve.
\end{abstract}

Objective To determine whether differences in metabolic paremeters exist in T1DM + CD compared to cohort of patients with T1DM attending the Diabetes Day Centre, Galway University Hospital

\section{Methods}

A Single-centre, cross-sectional study. All patients with T1DM attending the Diabetes Day Centre, GUH. Data collection via DIAMOND and PAS, for clinic attendances June 2011 to June 2013

Subgroup analysis for those with TIDM + CD: All patients have histology diagnosis. Compliance to GFD determined by Anti-tissue translglutaminase antibodies (anti-tTG) titre $<10 \mathrm{U} / \mathrm{ml}$

Metabolic parameters: Weight, BMI, Blood Pressure,HbAlC,Lipid profiles, Use of lipid lowering drugs were all collected at routine clinic visits

Statistical analysis: Minitab Version 16 Descriptive analysis. Student' s t-test

Results
-CD prevalence in T1DM attending Diabetes Day Centre,
GUH was (4.1\%)
-HbA1C in the T1DM + CD was statistically higher than T1DM,
(76.4mmol/mol \pm 17.4 vs $70.3 \pm 17.7)$
-HbA1C was higher in those with poor compliance to GFD
(66 \pm 13.1 vs $81.2 \pm 23.5)$
-CD patients tend to have better lipid profile and lower BMI
-*targets are those of the American Diabetes Association ${ }^{2}$
-* hypoparathyroidism, vitiligo, pernicious anaemia

Table 1: Comparison of demographic and metabolic parameters

\begin{tabular}{lcc}
\hline Variables & T1DM + CD & T1DM \\
\hline Patients, $\mathrm{n}(\%)$ & $37(4.1 \%)$ & $868(95.9 \%)$ \\
Male, $\mathrm{n}(\%)$ & $17(46 \%)$ & $457(52.6 \%)$ \\
Age $>18, \mathrm{n}(\%)$ & $32(86.4 \%)$ & $766(88.2 \%)$ \\
Mean age (year), \pm SD & $38.9 \pm 18$ & $37 \pm 17$ \\
\hline
\end{tabular}

\section{Metabolic Parameters}

$\mathrm{HbA} 1 \mathrm{C}(\mathrm{mmol} / \mathrm{mol}),($ mean $\pm \mathrm{SD})$
$<18 \mathrm{y} / \mathrm{o}$
$>18 \mathrm{y} / \mathrm{o}$

Lipid Profile*

Total cholesterol $(\mathrm{mmol} / \mathrm{L})$

(mean \pm SD)

In target*, n (\%)

Triglycerides $(\mathrm{mmol} / \mathrm{L}),($ mean $\pm \mathrm{SD})$

In target*, n (\%)

$\mathrm{LDL}(\mathrm{mmol} / \mathrm{L})$, mean $\pm \mathrm{SD}$

In target*, n (\%)

$\mathrm{HDL}(\mathrm{mmol} / \mathrm{L})$, mean \pm SD

In target*, n (\%)

Patients on lipid lowering drugs, $n(\%)$

Body Mass Index $\left(\mathrm{kg} / \mathrm{m}^{2}\right)($ mean $\pm \mathrm{SD})$

In target ${ }^{\ddagger}, \mathrm{n}(\%)$

$\begin{array}{ccc}76.4 \pm 17.4 & 70.3 \pm 17.7 & 0.04 \\ 83.6 \pm 12.8 & 75.7 \pm 15.7 & 0.25 \\ 72.8 \pm 21.4 & 69.6 \pm 17.8 & 0.41 \\ p=0.15 & p<0.05 & \\ & & \\ 4.0 \pm 1.0 & 4.6 \pm 1.1 & 0.001 \\ 32(87 \%) & 634(73 \%) & 0.85 \\ & & \\ 1.0 \pm 0.4 & 1.3 \pm 1.2 & 0.002 \\ 36(97 \%) & 712(82 \%) & 0.001 \\ & & \\ 2.0 \pm 0.8 & 2.4 \pm 0.8 & 0.003 \\ 32(87 \%) & 538(62 \%) & 0.003 \\ & & \\ 2.0 \pm 0.6 & 1.7 \pm 0.5 & 0.001 \\ 34(93 \%) & 738(85 \%) & 0.34\end{array}$

$34(93 \%) \quad 738(85 \%)-0.34$

$11(35 \%) \quad 269(31 \%) \quad N / S$
Table 2: T1DM+CD group description

\section{T1DM + CD}

Duration of disease (year), mean \pm SD
Anti-tTG (U/ml), mean \pm SD
Autoimmune disease
Hypothyroidism, $n(\%)$
Addison's disease, $n(\%)$
Others, $n(\%)$
Metformin, $n(\%)$
DAFNE, $n(\%)$
Insulin pump, $n(\%)$

$9.2 \pm 8.0$

$43 \pm 46.4$

$7(18.9 \%)$

0

$3(8.1 \%)$

0

$5(13.5 \%)$

$2(5.4 \%)$

Table 3: T1DM+CD gp compliant vs non-compliant to Gluten free diet

$$
\mathrm{T} 1 \mathrm{DM}+\mathrm{CD}
$$

\begin{tabular}{lccc} 
& Compliant & $\begin{array}{c}\text { Non- } \\
\text { compliant }\end{array}$ & p-value \\
\hline Patients, $\boldsymbol{n}(\%)$ & $17(45.9 \%)$ & $20(54.1 \%)$ & \\
Duration of disease (yrs) & $9.3 \pm 11.3$ & $9.1 \pm 4.12$ & 0.9 \\
Anti-tTG abs (U/ml) & $2.12 \pm 3.22$ & $65.1 \pm 50.2$ & $<0.005$ \\
Male, $\mathbf{n}(\%)$ & $7(41.2 \%)$ & $8(40 \%)$ & $\mathrm{N} / \mathrm{S}$ \\
BMI $\left(\mathrm{kg} / \mathrm{m}^{2}\right)$ & $23.1 \pm 4.63$ & $25.22 \pm 4.57$ & 0.2 \\
HbA1C $(\mathrm{mmol} / \mathrm{mol})$ & $66 \pm 13.1$ & $81.2 \pm 23.5$ & $\mathbf{0 . 0 2}$ \\
\hline
\end{tabular}

\section{Conclusions}

The management of T1D with co-morbid coeliac disease presents an extra challenge. Lipid profiles tend to be better, but overall the HbA1c is worse.

Those with poor compliance to GFD have better HbA1c suggesting that the diet makes achieving target HbA1c more difficult 